PROCEEDINGS OF THE

AMERICAN MATHEMATICAL SOCIETY

Volume 128, Number 8, Pages 2281-2285

S 0002-9939(00)05296-5

Article electronically published on February 21, 2000

\title{
AN ARITHMETIC OBSTRUCTION TO DIVISION ALGEBRA DECOMPOSABILITY
}

\author{
ERIC S. BRUSSEL
}

(Communicated by Ken Goodearl)

\begin{abstract}
This paper presents an indecomposable finite-dimensional division algebra of $p$-power index that decomposes over a prime-to- $p$ degree field extension, obtained by adjoining $p$-th roots of unity to the base. This shows that the theory of decomposability has an arithmetic aspect.
\end{abstract}

Suppose $F$ is a field and $D$ is an indecomposable $F$-division algebra, that is, a division algebra that cannot be expressed as the tensor product of two nontrivial $F$-division algebras. It is easy to see that the (Schur) index of $D$ must be a power of some prime $p$. In "Problem 6" of [Sa], Saltman asks if in general $D$ remains indecomposable upon arbitrary prime-to- $p$ extension. At issue is the nature of indecomposability, in particular whether or not it is "geometric". For example in [K], Karpenko showed a certain generic class of division algebras are indecomposable by computing the degrees of cycles on their Brauer-Severi varieties. As noted in $[\mathrm{K}$, it is immediate from the geometric nature of the proof that these algebras remain indecomposable over all prime-to- $p$ extensions.

This paper presents an indecomposable division algebra that decomposes over a prime-to- $p$ extension, namely the cyclotomic extension defined by $p$-th roots of unity. Thus it is proved that (in)decomposability can have an arithmetic aspect.

Let $p$ be an odd prime of $\mathbb{Q}$, let $k$ be a number field that does not contain a $p^{t h}$ root of unity, and let $k[s, t]$ be the polynomial ring in two variables over $k$. Define

$$
\begin{aligned}
v: k[s, t] & \rightarrow \mathbb{Z} \oplus \mathbb{Z}, \\
f & \mapsto(a, b)
\end{aligned}
$$

where $b$ is smallest such that $f \in\left(t^{b}\right)$ and $a$ is smallest such that $f \in\left(s^{a}, t^{b+1}\right)$. The map $v$ is a valuation, with value group $\mathbb{Z} \oplus \mathbb{Z}$ ordered reverse lexicographically, so $(a, b)<\left(a^{\prime}, b^{\prime}\right)$ if $b<b^{\prime}$, or if $b=b^{\prime}$ and $a<a^{\prime}$. The field of iterated power series

$$
F=k((s))((t))
$$

is Henselian with respect to $v$, with valuation ring

$$
R=k[[s]]+t k((s))[[t]] \subset k((s))[[t]] .
$$

$R$ is a non-Noetherian 2-dimensional Henselian local ring, with maximal ideal $(s)=$ $(s, t)$ and residue field $k$. The ideal $(s)$ properly contains the (infinitely generated) prime ideal $t k((s))[[t]]=\left(t, \frac{t}{s}, \frac{t}{s^{2}}, \ldots\right)$.

Received by the editors June 10, 1998 and, in revised form, October 6, 1998.

1991 Mathematics Subject Classification. Primary 16K20; Secondary 11R37.

(C)2000 American Mathematical Society 
For any field $l$, let $\mathrm{X}(l)$ denote the character group of $l$, consisting of all continuous homomorphisms from the Galois group $G_{l}$ to the group of roots of unity $\mu(\mathbb{C})$. If $\xi \in \mathrm{X}(l)$, let $l(\xi) / l$ denote the cyclic extension (of degree $|\xi|$ ) determined by $\xi$. Let $\langle\psi, \theta\rangle \subset \mathrm{X}(l)$ denote the subgroup determined by $\psi$ and $\theta$. If $G \subset \mathrm{X}(l)$ is any subgroup, let $l(G)$ denote the composite of the extensions determined by the elements of $G$.

Let $\mu_{n}$ denote the $n$-th roots of unity in $\mathbb{C}$. By Kummer theory if $\mu_{n} \subset l^{\bullet}$, there is an isomorphism

$$
l / l^{\bullet} \stackrel{\sim}{\sim} \mathrm{X}(l)_{n}
$$

where $\mathrm{X}(l)_{n}$ denotes the $n$-torsion of $\mathrm{X}(l)$. If $\xi \in \mathrm{X}(l)_{n}$ is represented by $u \bmod l^{\bullet}$, then $k(\xi) \cong l\left(u^{1 / n}\right)$.

In the Brauer group $\operatorname{Br}(l)$ let $(\xi, t)$ denote the cyclic element determined by character $\xi$ and element $t \in l^{*}$. If $\mu_{n} \subset l$ and $\xi \in \mathrm{X}(l)_{n}$ is represented by $u \bmod l^{*}$, then $(\xi, t)=(u, t)_{n}$, the symbol defined by $u$ and $t$.

There is an exact sequence

$$
0 \rightarrow \operatorname{Br}(k) \rightarrow \operatorname{Br}(F) \stackrel{T}{\rightarrow} \mathrm{X}(k((s))) \coprod \mathrm{X}(k((t))) \stackrel{\text { ord }}{\longrightarrow} \mu(k) \rightarrow 0 .
$$

The maps: $\operatorname{Br}(k) \rightarrow \operatorname{Br}(F)$ is the usual restriction. $T$ is the sum of the two residue maps corresponding to the discrete valuations $t$ (on $F=k((s))((t))$ ) and $s$ (on $k((t))((s)))$. The natural isomorphism $\operatorname{Br}(k((s))((t))) \cong \operatorname{Br}(k((t))((s)))$ shows both are defined on $\operatorname{Br}(F)$. Finally, ord is the ramification with respect to the valuation $v$.

Exactness of (1) is proved in [B] by iteratively applying Witt's theorem ( $\mathrm{Se}]$, which describes the Brauer group of discretely Henselian fields with perfect residue field. Briefly, the kernel of $T$ consists of the $v$-unramified elements $\alpha$ of $\operatorname{Br}(F)$, and since $R$ is Henselian this is $\operatorname{Br}(k)$. The residue maps are separately surjective, and thus the image of $T$ and the kernel of ord both consist of elements of the form $\left(\psi+s^{m / n}, \theta+t^{-m / n}\right)$ where $\psi, \theta \in \mathrm{X}(k), n=|\mu(k)|, m \in\{0,1, \ldots, n-1\}$, and $s^{m / n}$ and $t^{-m / n}$ stand for the characters they determine under the Kummer map.

By Witt's theorem, (1) "splits", so that any $\delta \in \operatorname{Br}(F)$ has the form

$$
\delta=\alpha+(\psi, s)+(\theta, t)+m(s, t)_{n}
$$

with $\alpha \in \operatorname{Br}(k), \psi, \theta \in \mathrm{X}(k), n=|\mu(k)|$, and $m \in\{0,1, \ldots, n-1\}$.

In the following write $D(\delta)$ for the division algebra underlying a Brauer element $\delta$. Write $\operatorname{ind}(D)$ and $\operatorname{per}(D)$ for the index and period of $D$, respectively. Assume always that $p$ is an odd prime and $\mu_{p} \not \subset F^{\circ}$. Then $\mu(k)(p)$ is trivial and so the symbol term in (2) is trivial. More generally, for all $\delta \in \operatorname{Br}(F)$ of the form $\delta=$ $\alpha+(\psi, s)+(\theta, t)$, the index formula is

$$
\operatorname{ind}(\delta)=|G| \operatorname{ind}\left(\alpha^{k(G)}\right)
$$

where $G=\langle\psi, \theta\rangle$ and $\alpha^{k(G)}$ is the restriction of $\alpha$ to $\operatorname{Br}(k(G))$. This and a more general index formula are proved by iteratively applying Nakayama's index formula for discretely Henselian fields $([\underline{B}]$ ).

Theorem. Let $p$ be an odd prime, $k$ a number field not containing $\mu_{p}$, and let $F$ be the twice iterated power series field above. Then there exists an indecomposable $F$-division algebra $D$ of period $p^{4}$ and index $p^{5}$ that becomes decomposable over the prime-to-p extension $k\left(\mu_{p}\right)$. 
Proof. Select three primes $\mathfrak{q}, \mathfrak{q}^{\prime}$, and $\mathfrak{p}$ of $k$, such that:

$$
\begin{gathered}
\mu_{p^{2}} \subset k_{\mathfrak{q}}, k_{\mathfrak{q}^{\prime}} \\
\mu_{p} \not \subset k_{\mathfrak{p}} .
\end{gathered}
$$

Let $\psi_{\mathfrak{q}}$ and $\psi_{\mathfrak{q}^{\prime}}$ be totally ramified (local) characters of order $p^{2}$, let $\theta_{\mathfrak{q}}$ and $\theta_{\mathfrak{q}^{\prime}}$ be unramified of order $p^{2}$, let $\psi_{\mathfrak{p}}$ be trivial, and let $\theta_{\mathfrak{p}}$ be unramified of order $p$. By Grunwald-Wang's Theorem ([AT] $)$, there exist (global) characters $\psi$ and $\theta$ with

$$
|\psi|=|\theta|=p^{2}
$$

and with the above restrictions at $\mathfrak{p}, \mathfrak{q}$, and $\mathfrak{q}^{\prime}$. Set $G=\langle\psi, \theta\rangle$. The groups $\left\langle\psi_{\mathfrak{q}}\right\rangle$ and $\left\langle\theta_{\mathfrak{q}}\right\rangle$ are disjoint in $\mathrm{X}\left(k_{\mathfrak{q}}\right)$, so $\left|G_{\mathfrak{q}}\right|=p^{4}$, and similarly $\left|G_{\mathfrak{q}^{\prime}}\right|=p^{4}$. Therefore $\langle\psi\rangle$ and $\langle\theta\rangle$ are disjoint in $\mathrm{X}(k)$, and $|G|=p^{4}$. Let $\alpha$ be the unramified element of $\operatorname{Br}(F)$ with invariants

$$
\begin{aligned}
\operatorname{inv}\left(\alpha_{\mathfrak{q}}\right) & =1 / p^{4} \\
\operatorname{inv}\left(\alpha_{\mathfrak{p}}\right) & =1 / p^{2} \\
\operatorname{inv}\left(\alpha_{\mathfrak{q}^{\prime}}\right) & =1-\operatorname{inv}\left(\alpha_{\mathfrak{q}}\right)-\operatorname{inv}\left(\alpha_{\mathfrak{p}}\right) .
\end{aligned}
$$

Let

$$
D=D(\alpha+(\psi, s)+(\theta, t))
$$

as per (2). By direct computation,

$$
\begin{aligned}
& \operatorname{ind}(D)=|G| \cdot \operatorname{ind}\left(\alpha^{K(G)}\right)=p^{4} \cdot p=p^{5}, \\
& \operatorname{per}(D)=\operatorname{lcm}\{\operatorname{ind}(\alpha),|\psi|,|\theta|\}=p^{4} .
\end{aligned}
$$

The index follows since $|G|=p^{4}$ and $k(G)$ splits $\alpha$ at every prime except $\mathfrak{p}$, while $\operatorname{ind}\left(\alpha_{\mathfrak{p}}^{k\left(G_{\mathfrak{p}}\right)}\right)=p$. The period uses (1) and the fact that period equals index in $\operatorname{Br}(k)$.

Claim 1. $D$ is indecomposable. Suppose not; let

$$
D \cong D_{1} \otimes D_{2}
$$

be a nontrivial decomposition. By dimension count $\operatorname{ind}(D)=\operatorname{ind}\left(D_{1}\right) \operatorname{ind}\left(D_{2}\right)$. In the following, let the subscripts "1" and "2" signify association with $D_{1}$ and $D_{2}$. By (3),

$$
|G| \operatorname{ind}\left(\alpha^{k(G)}\right)=\left|G_{1}\right| \operatorname{ind}\left(\alpha_{1}^{k\left(G_{1}\right)}\right) \cdot\left|G_{2}\right| \operatorname{ind}\left(\alpha_{2}^{k\left(G_{2}\right)}\right) .
$$

Since $\psi=\psi_{1}+\psi_{2}$ and $\theta=\theta_{1}+\theta_{2}, G \subseteq G_{1} G_{2}$.

Assume without loss of generality that $\operatorname{ind}\left(D_{1}\right) \leq \operatorname{ind}\left(D_{2}\right)$. Then $\operatorname{ind}\left(D_{1}\right)=p^{2}$ or $p$, and $\operatorname{ind}\left(D_{2}\right)=p^{3}$ or $p^{4}$. By (3), $\left|G_{1}\right|$ divides $p^{2}$, and since $|G|=p^{4}$ and $G \subseteq G_{1} G_{2}, p^{2}$ divides $\left|G_{2}\right|$. Since $\alpha=\alpha_{1}+\alpha_{2}, \operatorname{per}(\alpha)=p^{4}$, and $\operatorname{per}\left(\alpha_{1}\right)$ divides $p^{2}$, by abelian group theory $\operatorname{per}\left(\alpha_{2}\right)=p^{4}$. Therefore $\operatorname{per}\left(D_{2}\right)=p^{4}$; hence $\operatorname{ind}\left(D_{2}\right)=p^{4}$, and this forces $\operatorname{ind}\left(D_{1}\right)=p$. It follows that

$$
\begin{aligned}
& \left|G_{1}\right| \text { divides } p, \\
& \left|G_{2}\right|=p^{2}, p^{3} \text {, or } p^{4} .
\end{aligned}
$$

If $\left|G_{2}\right|=p^{2}$, then since $G_{1} G_{2} \supseteq G$ and $|G|=p^{4}$, necessarily $\left|G_{1}\right|=p^{2}$, which is not the case. If $\left|G_{2}\right|=p^{3}$, then again $p^{2}$ divides $\left|G_{1}\right|$, because $G_{1} G_{2}$ must contain the disjoint cyclic groups $\langle\theta\rangle$ and $\langle\psi\rangle$ each of which has order $p^{2}$. Therefore it must be that $\left|G_{2}\right|=p^{4}$, and since $\operatorname{ind}\left(D_{2}\right)=p^{4}, \operatorname{ind}\left(\alpha_{2}^{k\left(G_{2}\right)}\right)=1$. If $G_{1}$ is 
trivial, then $G_{2}=G$, and $G$ does not split the invariants of $\alpha_{2}$ at $\mathfrak{q}$, contradicting $\operatorname{ind}\left(\alpha_{2}^{k\left(G_{2}\right)}\right)=1$. Therefore $\left|G_{1}\right|=p$ and $\operatorname{ind}\left(\alpha_{1}^{k\left(G_{1}\right)}\right)=1$.

Since $G_{2}$ is abelian, $\left(G_{2}\right)_{\mathfrak{p}}$ is abelian, and since $\mu_{p} \not \subset k_{\mathfrak{p}},\left(G_{2}\right)_{\mathfrak{p}}$ is cyclic. By abelian group theory $\operatorname{ind}\left(\left(\alpha_{2}\right)_{\mathfrak{p}}\right)=\operatorname{ind}\left(\alpha_{\mathfrak{p}}\right)=p^{2}$, since $\operatorname{ind}\left(\left(\alpha_{1}\right)_{\mathfrak{p}}\right) \mid p$. Since $G_{2}$ splits $\alpha_{2}, p^{2}$ divides $\left|\left(G_{2}\right)_{\mathfrak{p}}\right|$; hence $p^{2}$ divides the group exponent of $\left(G_{2}\right)_{\mathfrak{p}}$. But $\left(G_{2}\right)_{\mathfrak{p}} \subseteq G_{\mathfrak{p}}\left(G_{1}\right)_{\mathfrak{p}}$, and the right side has group exponent $p$. This is a contradiction, proving claim 1 .

Claim 2. $D\left(D \otimes F\left(\mu_{p}\right)\right)$ is decomposable. This will be proved by a construction over $F\left(\mu_{p}\right)$. Since $F\left(\mu_{p}\right) / F$ has prime-to- $p$ degree, the orders, degrees, and ramification behavior of the objects associated to $D$ do not change from $D$ to $D \otimes F\left(\mu_{p}\right)$. In the following, identify $\mathfrak{p}, \mathfrak{q}$, and $\mathfrak{q}^{\prime}$ with chosen extensions to $k\left(\mu_{p}\right)$.

Let $\varphi_{\mathfrak{p}} \in \mathrm{X}\left(k\left(\mu_{p}\right)_{\mathfrak{p}}\right)$ be totally ramified of order $p$ (existence requires the root of unity), and let $\varphi_{\mathfrak{q}}$ and $\varphi_{\mathfrak{q}^{\prime}}$ both be trivial. Let $\varphi \in \mathrm{X}\left(k\left(\mu_{p}\right)\right)$ be a character of order $p$ with these restrictions. Note that $\theta_{\mathfrak{p}}$ and $\varphi_{\mathfrak{p}}$ are disjoint over $k\left(\mu_{p}\right)_{\mathfrak{p}}$, whereas there is only the unramified character over $k_{\mathfrak{p}}$, since $\mu_{p} \not \subset k_{\mathfrak{p}}$. Set

$$
\begin{aligned}
\psi_{1} & =\theta_{1}=\varphi, \\
\psi_{2} & =\psi^{F\left(\mu_{p}\right)}-\varphi, \\
\theta_{2} & =\theta^{F\left(\mu_{p}\right)}-\varphi, \\
\alpha_{2} & =\alpha^{F\left(\mu_{p}\right)} .
\end{aligned}
$$

Then set $D_{1}=D\left(\left(\psi_{1}, s\right)+\left(\theta_{1}, t\right)\right)$ and $D_{2}=D\left(\alpha_{2}+\left(\psi_{2}, s\right)+\left(\theta_{2}, t\right)\right)$.

A simple check that $\psi^{F\left(\mu_{p}\right)}=\psi_{1}+\psi_{2}$ and $\theta^{F\left(\mu_{p}\right)}=\theta_{1}+\theta_{2}$ shows that $D \otimes F\left(\mu_{p}\right) \sim$ $D_{1} \otimes D_{2}$. To prove $D\left(D \otimes F\left(\mu_{p}\right)\right) \cong D_{1} \otimes D_{2}$ it remains to show that the indexes are multiplicative. The index of $D_{1}$ is

$$
\operatorname{ind}\left(D_{1}\right)=\left|G_{1}\right|=\left|\left\langle\psi_{1}, \theta_{1}\right\rangle\right|=|\langle\varphi\rangle|=p .
$$

The order of $G_{2}=\left\langle\psi_{2}, \theta_{2}\right\rangle$ is $p^{4}$ : For

$$
\left\langle G_{2}, \varphi\right\rangle=\left\langle G^{F\left(\mu_{p}\right)}, \varphi\right\rangle=\left\langle\psi^{F\left(\mu_{p}\right)}\right\rangle, \theta^{F\left(\mu_{p}\right)}, \varphi,
$$

and since $\varphi_{\mathfrak{p}} \notin\left\langle\theta_{\mathfrak{p}}, \psi_{\mathfrak{p}}\right\rangle=\left\langle\theta_{\mathfrak{p}}\right\rangle, \varphi \notin G^{F\left(\mu_{p}\right)}$; hence $\varphi \notin G_{2}$ (else $G_{2}$ is a 3 generator group). Therefore $p \cdot\left|G_{2}\right|=p \cdot|G|$; hence $\left|G_{2}\right|=|G|=p^{4}$. Now compute $\operatorname{ind}\left(\alpha_{2}^{k\left(G_{2}\right)}\right)$ : At $\mathfrak{q}$ and $\mathfrak{q}^{\prime}, G_{1}$ is trivial, so $\left|\left(G_{2}\right)_{\mathfrak{q}}\right|=\left|G_{\mathfrak{q}}\right|=p^{4}$ and $\left|\left(G_{2}\right)_{\mathfrak{q}^{\prime}}\right|=\left|G_{\mathfrak{q}^{\prime}}\right|=p^{4}$. At $\mathfrak{p}, G_{2}$ is the noncyclic group $\left\langle\theta^{F\left(\mu_{p}\right)}\right\rangle_{\mathfrak{p}}, \varphi_{\mathfrak{p}}$, so $\left|\left(G_{2}\right)_{\mathfrak{p}}\right|=p^{2}$. Therefore, by construction, $G_{2}$ splits $\alpha_{2}$ at each prime in the locus of $\alpha_{2}$, so $\operatorname{ind}\left(\alpha_{2}^{k\left(G_{2}\right)}\right)=1$. Since $\left|G_{2}\right|=p^{4}$, and $\operatorname{ind}\left(\alpha_{2}^{k\left(G_{2}\right)}\right)=1$,

$$
\operatorname{ind}\left(D_{2}\right)=p^{4} \text {. }
$$

Therefore $\operatorname{ind}\left(D \otimes F\left(\mu_{p}\right)\right)=\operatorname{ind}\left(D_{1}\right) \operatorname{ind}\left(D_{2}\right)=p^{5}$, and so $D\left(D \otimes F\left(\mu_{p}\right)\right)$ is decomposable. This proves claim 2 , hence the theorem.

Remark. A general criterion for decomposability over $F$ is given in $[\mathrm{B}]$.

\section{REFERENCES}

[AT] Artin, E., Tate, J.: Class Field Theory, Addison-Wesley, Reading, Mass., 1967. MR 36:6383

[B] Brussel, E.: Division algebras over Henselian fields of rank two, (in preparation).

[K] Karpenko, N.: Torsion in $\mathrm{CH}^{2}$ of Severi-Brauer varieties and indecomposability of generic algebras, Manuscripta Math. 88 (1995), 109-117. MR 96g:14007 
[Sa] Saltman, D.: Finite dimensional division algebras. Azumaya Algebras, Actions, and Modules, (D. Haile and J. Osterburg, eds.), Contemporary Math. Vol. 124, Amer. Math. Soc., Providence, R.I., 1992, 203-214. MR 93a:16014

[Se] Serre, J.-P: Local Fields, Springer Verlag, New York, 1979. MR 82e:12016

Department of Mathematics and Computer Science, Emory University, Atlanta, GeorGIA 30322

E-mail address: brussel@mathcs.emory.edu 